\title{
Nuevo fenotipo de la enfermedad de Pompe infantil
}

\author{
Andrés Nascimento, Enrique Villalobos-Pinto
}

Introducción. La enfermedad de Pompe infantil es una glucogenosis por deficiencia de la enzima alfa-glucosidasa ácida. Antes de disponer del tratamiento enzimático sustitutivo (TES) específico, la forma clásica era mortal antes de los 2 años de vida. El TES aumenta la supervivencia y mejora la función cardíaca, respiratoria y motora.

Casos clínicos. Caso 1: lactante de 2 meses con hipotonía de predominio axial y portadora de sonda nasogástrica por dificultades en la succión y la deglución. Se evidenció miopatía y miocardiopatía hipertrófica. Se diagnosticó enfermedad de Pompe, se inició TES y se observó una mejoría de la función cardíaca y motora. Sin embargo, presentó infecciones respiratorias recurrentes que finalmente obligaron a una traqueostomía. Actualmente continúa con TES, camina con un andador y presenta una disfunción ventricular leve. Caso 2: lactante de 3 semanas que acudió a revisión rutinaria por su pediatra. En la exploración se apreció un soplo sistólico e hipotonía axial y proximal. En las pruebas cardiológicas se evidenció una miocardiopatía hipertrófica. Se envió al paciente a un centro de referencia donde se diagnosticó enfermedad de Pompe y un estado del material inmunológico con reactividad cruzada negativo. El paciente recibió tratamiento inmunomodulador y TES. La evolución fue favorable, aunque presentó infecciones respiratorias frecuentes. En la actualidad ha conseguido la deambulación autónoma, pero la marcha es inestable.

Conclusiones. Ambos casos ilustran el nuevo fenotipo de la enfermedad de Pompe infantil tratada con TES. A pesar de las limitaciones motoras y la afectación respiratoria que presentan los pacientes, la supervivencia y la autonomía han aumentado.

Palabras clave. Alfa-glucosidasa ácida. Enfermedad de Pompe. Estado CRIM. Hipotonía. Miocardiopatía hipertrófica. Tratamiento enzimático sustitutivo.

\section{Introducción}

La enfermedad de Pompe (OMIM n. $\left.{ }^{\circ} 232300\right)$, también conocida como glucogenosis de tipo II o deficiencia de maltasa ácida, es un trastorno metabólico multisistémico, de herencia autosómica recesiva y raro. Está causada por mutaciones del gen que codifica la enzima $\alpha$-glucosidasa ácida (GAA). Debido a la deficiencia de GAA, se produce un almacenamiento excesivo de glucógeno en los lisosomas, lo que provoca disfunción celular y daño progresivo de la musculatura cardíaca, respiratoria, esquelética y lisa [1]. Sus manifestaciones clínicas y su evolución son muy variables [2], incluso en pacientes con la misma mutación, lo que indica la influencia de otros factores modificadores del fenotipo de la enfermedad [3].

Pueden distinguirse dos formas: la infantil o de inicio precoz y la de inicio tardío. La actividad de la GAA suele ser inferior al $1 \%$ en la enfermedad de Pompe infantil, pero se conserva hasta un $40 \%$ en la forma de inicio tardío [2]. La forma infantil clásica, descrita por Pompe [4], es la más grave. De inicio en el primer año de vida, puede cursar con miocardiopatía, afectación respiratoria, hipotonía, debilidad muscular, dificultades en la deglución y hepatomegalia. La enfermedad se manifiesta antes de los 12 meses de vida, generalmente hacia el mes y medio o los 2 meses, y progresa con rapidez [5]. Antes de la aparición del tratamiento enzimático sustitutivo (TES) específico, los pacientes morían por complicaciones cardíacas o respiratorias antes de los 2 años de edad [6]. Existe una forma clásica atípica sin miocardiopatía y de evolución más lenta [7].

El manejo de los pacientes con enfermedad de Pompe infantil requiere un equipo multidisciplinar y bien coordinado. Actualmente se dispone de una guía española para el diagnóstico, el tratamiento y el seguimiento de estos pacientes. Esta guía incluye una descripción de las manifestaciones clínicas y una serie de recomendaciones para el diagnóstico, el manejo por especialidades y el uso del TES, así como un cronograma para el seguimiento de los pacientes [7].

El diagnóstico de la enfermedad de Pompe infantil se realiza a partir de la sospecha clínica. En
Hospital Universitari Sant Joan de Déu; U.B. CIBER; Instituto de Salud Carlos III; Esplugues de Llobregat, Barcelona (A. Nascimento). Servicio de Pediatría; Hospital Infantil Universitario Niño Jesús; Madrid, España (E. Villalobos-Pinto).

Correspondencia:

Dr. Enrique Villalobos Pinto. Servicio de Pediatría. Hospital Infantil Universitario Niño Jesús. Avda. Menéndez Pelayo, 65. E-28009 Madrid.

E-mail: evillalobospinto@gmail.com

Agradecimientos:

Content Ed Net, por su asistencia editorial en la publicación de este manuscrito.

Aceptado tras revisión externa: 11.01.18.

Cómo citar este artículo: Nascimento A, Villalobos-Pinto E. Nuevo fenotipo de la enfermedad de Pompe infantil. Rev Neurol 2018; 66: 121-4.

(C) 2018 Revista de Neurología 
primer lugar, se realiza la prueba de la gota de sangre seca, que es sencilla y orienta sobre el grado de actividad de la GAA. El resultado de dicha prueba debe confirmarse con la determinación de la actividad enzimática en linfocitos, leucocitos, músculos o fibroblastos. Por último, es importante el estudio de las mutaciones genéticas [7].

En cuanto al tratamiento, el TES con GAA recombinante humana ha modificado el pronóstico de la enfermedad de Pompe infantil [6]. Aumenta la supervivencia y mejora la función cardíaca, respiratoria y motora. El diagnóstico precoz es esencial porque cuanto más temprano sea el inicio del TES, mayores son los beneficios [7]. Sin embargo, antes de iniciar el TES debe determinarse el estado del material inmunológico con reactividad cruzada (CRIM), que corresponde a la GAA residual. Hasta un 32\% de los pacientes son CRIM negativos, es decir, carecen de GAA residual y producen anticuerpos contra la GAA recombinante humana. Cuanto mayor sea la formación de anticuerpos, menor es la respuesta al TES y peor es el pronóstico. No obstante, la administración de un tratamiento inmunomodulador puede mejorar el pronóstico de los pacientes CRIM negativos [8]. La pauta más habitual consiste en rituximab, metotrexato e inmunoglobulina inmediatamente antes de la administración del TES [9].

\section{Casos clínicos}

\section{Caso 1}

Lactante de 2 meses de edad que consultó por hipotonía y dificultad en la succión y la deglución que requería alimentación por sonda nasogástrica, sin antecedentes familiares ni obstétricos de interés. En la exploración física se constató un estado nutricional y de hidratación dentro de la normalidad, buen contacto y seguimiento visual, y auscultación cardiorrespiratoria normal. Sin embargo, la paciente mostraba hipotonía de predominio axial e hiporreflexia. La analítica general fue anodina.

Ante la sospecha de una enfermedad neuromuscular, se realizó una electromiografía, que mostró trazados polifásicos de baja amplitud, abundante actividad espontánea en forma de fibrilación y ondas positivas y descargas de alta frecuencia. Se pidieron enzimas musculares y se halló una creatincinasa de $350 \mathrm{UI} / \mathrm{L}$ (normal: < $190 \mathrm{UI} / \mathrm{L}$ ).

Ante estos datos, se indicó un estudio cardiológico. En el electrocardiograma se observó un intervalo P-R corto. En la ecocardiografía, la fracción de eyección era del $45 \%$, y el ventrículo izquierdo esta- ba engrosado, con una pared posterior de $8 \mathrm{~mm}$ y un septo interventricular de $10 \mathrm{~mm}$. Se dictaminó que la paciente presentaba una miocardiopatía hipertrófica concéntrica no obstructiva.

La coexistencia de miocardiopatía, enfermedad muscular y trastorno de la deglución sugería una enfermedad de Pompe infantil. La prueba de la gota de sangre seca y la actividad de la GAA en los leucocitos confirmaron el diagnóstico, con una actividad enzimática $<1 \%$. El estudio genético mostró dos mutaciones patógenas en el gen de la GAA. Debido a la gravedad del cuadro clínico, se decidió iniciar TES (20 mg/kg cada 2 semanas por vía intravenosa) a los 2 meses de edad sin esperar los resultados del estado CRIM. No se realizó inmunoterapia, ya que en ese momento no estaba establecido el protocolo. La paciente toleró bien el TES. La miocardiopatía hipertrófica mejoró, con una fracción de eyección del 55\% y una reducción del grosor de la pared ventricular (pared posterior del ventrículo izquierdo de $7 \mathrm{~mm}$ y septo interventricular de $7 \mathrm{~mm}$ ) a los 12 meses de vida. A los 10 meses, la paciente se mantenía sentada sin apoyo. A los 18 meses, la paciente caminaba y se levantaba del suelo sin ayuda. Sin embargo, continuaba con dificultades en la deglución y presentaba infecciones respiratorias recurrentes.

A los 3 años, ingresó en la unidad de cuidados intensivos por insuficiencia respiratoria durante un episodio infeccioso. Se inició ventilación no invasiva, pero no la toleró y precisó ventilación mecánica. A las 3 semanas con ventilación mecánica, la paciente presentó hipotonía intensa, muy escasa movilidad de las extremidades, ausencia de movilidad diafragmática y pérdida de la capacidad de control cefálico, sedestación y bipedestación. Se practicó una traqueostomía y se mantuvo el TES. Una vez estabilizada la paciente, se realizó un estudio de deglución con videofluoroscopia que mostró una disfagia moderada-grave, por lo que se practicó una gastrostomía. La paciente no requirió nuevas hospitalizaciones por infecciones respiratorias.

A los 4 años, los padres refirieron falta de atención y disminución de la respuesta a estímulos auditivos desde hacía unos 6 meses. Se realizó un estudio auditivo completo con estudio de potenciales evocados auditivos del tronco cerebral, timpanometría y audiometría. Se determinó que la paciente presentaba una hipoacusia de transmisión con timpanometría plana, que mejoró con tratamiento sintomático.

Actualmente la paciente tiene 5 años y sigue en tratamiento con TES con la misma pauta. El TES es bien tolerado, con anticuerpos negativos. Se man- 
tiene en pie sin apoyo y camina con un andador, aunque la debilidad muscular es generalizada. Cabe destacar que la paciente presenta afectación significativa de la musculatura flexora de los pies, por lo que precisa ortesis para mejorar el apoyo y facilitar la deambulación asistida. La debilidad de la musculatura anterior de la pierna con afectación del músculo tibial anterior es un patrón frecuente en los pacientes con formas clásicas (de inicio infantil) de la enfermedad de Pompe que reciben TES. En cambio, este patrón no se observa en los pacientes con formas de inicio tardío tratados o no con TES. La debilidad distal de la musculatura anterior de la pierna es una característica de las formas infantiles tratadas con TES y se considera como parte del nuevo genotipo en los pacientes tratados con TES.

La paciente continúa con alimentación por gastrostomía y requiere ventilación no invasiva nocturna con presión positiva (BiPAP) a través de la traqueostomía. Presenta una disfunción ventricular leve (fracción de eyección del 50\%) y taquicardia paroxística supraventricular secundaria a un síndrome de Wolf-Parkinson-White. Muestra un retraso en el desarrollo del lenguaje expresivo, probablemente debido a la hipoacusia, pero interacciona correctamente con su entorno social y familiar.

\section{Caso 2}

Lactante de 3 semanas que acudió a revisión rutinaria por su pediatra. Era hijo de padres consanguíneos. El embarazo y el parto fueron normales, así como el peso y la talla al nacer.

En la exploración física se apreciaron un soplo sistólico 3/6 multifocal e hipotonía axial y proximal. En el diagnóstico diferencial se contemplaron el hipotiroidismo, la glucogenosis de tipo II y las enfermedades peroxisómicas. Se remitió al paciente a cardiología, donde se observó una cardiomegalia en la radiografía de tórax. Se solicitó entonces una ecocardiografía, en la que se confirmó una miocardiopatía hipertrófica, con hipertrofia biventricular concéntrica. Ante la sospecha de una enfermedad de depósito, se derivó inmediatamente al paciente a un hospital de tercer nivel con experiencia en este tipo de enfermedades.

En el centro de referencia, la prueba de la gota de sangre seca indicó una actividad de GAA inferior al 1\%. A continuación se determinó la actividad enzimática en leucocitos, que confirmó el déficit enzimático y, por lo tanto, el diagnóstico de enfermedad de Pompe. El estudio genético halló una mutación en heterocigosis en el gen de la GAA. A continuación, se investigó el estado CRIM, que era nega- tivo. En consecuencia, antes de la primera dosis de TES se empezó a administrar un tratamiento inmunomodulador con rituximab, metotrexato e inmunoglobulinas según la pauta descrita por Banugaria et al [10]. Se inició TES $(20 \mathrm{mg} / \mathrm{kg} / \mathrm{dos}$ semanas por vía intravenosa) a las 6 semanas de vida. El paciente evolucionó favorablemente, aunque presentó infecciones respiratorias frecuentes.

En la actualidad, el paciente tiene 23 meses y continúa en tratamiento con TES con la misma pauta. Precisa ventilación no invasiva con BiPAP por las noches durante los episodios de infección respiratoria. Presenta asma intermitente con exacerbaciones frecuentes, y recibe corticoesteroides inhalados como tratamiento de mantenimiento y salbutamol inhalado en las exacerbaciones. Recibe también fisioterapia respiratoria. En cuanto a la afectación muscular, ha conseguido la deambulación autónoma, aunque la marcha es inestable y persiste la hipotonía.

\section{Discusión}

Se presentan dos casos de enfermedad de Pompe clásica. En el caso 1, la hipotonía y la dificultad en la deglución, asociadas a la miocardiopatía, debían haber orientado el diagnóstico hacia esta enfermedad desde un primer momento. En el caso 2, la enfermedad fue detectada en la revisión rutinaria por el pediatra. Es importante recordar que, ante un lactante hipotónico con cardiomegalia en la radiografía de tórax, debe sospecharse una enfermedad de Pompe [11].

Ambos casos ilustran el nuevo fenotipo de la enfermedad de Pompe infantil. El TES ha aumentado la supervivencia en los pacientes con esta enfermedad, pero, debido a la gravedad de la afectación cardíaca, respiratoria, motora y deglutoria, no siempre mejora significativamente la calidad de vida y la independencia motora [7]. Además, se ha observado que los pacientes tratados pueden presentar debilidad residual en algunos grupos motores, dificultades en la deglución y problemas en el manejo de las secreciones. Por ejemplo, las alteraciones respiratorias son frecuentes en los pacientes tratados con TES y se deben a debilidad progresiva de la musculatura respiratoria. No debe interrumpirse el TES durante los episodios respiratorios [7]. Otra complicación es la hipoacusia, como la presentada por el caso 1.

El TES ha mejorado la supervivencia y la función cardíaca y motora de los pacientes con enfermedad de Pompe infantil. Sin embargo, estos pacientes pue- 
den presentar diversas complicaciones que han de ser reconocidas y tratadas con un enfoque multidisciplinar por equipos médicos con experiencia en enfermedades neuromusculares.

\section{Bibliografía}

1. Kishnani PS, Amartino HM, Lindberg C, Miller TM, Wilson A, Keutzer J. Timing of diagnosis of patients with Pompe disease: data from the Pompe registry. Am J Med Genet A 2013; 161: 2431-43.

2. Van der Ploeg AT, Reuser AJ. Pompe's disease. Lancet 2008; 372: 1342-53.

3. De Filippi P, Saeidi K, Ravaglia S, Dardis A, Angelini C, Mongini T, et al. Genotype-phenotype correlation in Pompe disease, a step forward. Orphanet J Rare Dis 2014; 9: 102

4. Pompe JC. Over idiopathische hypertrophie van het hart. Ned Tijdschr Geneeskd 1932; 76: 304-12.

5. Kishnani PS, Hwu WL, Mandel H, Nicolino M, Yong F, Corzo D. A retrospective, multinational, multicenter study on the natural history of infantile-onset Pompe disease. J Pediatr 2006; 148: 671-6.
6. Kishnani PS, Beckemeyer AA, Mendelsohn NJ. The new era of Pompe disease: advances in the detection, understanding of the phenotypic spectrum, pathophysiology, and management. Am J Med Genet C Semin Med Genet 2012; 160C: 1-7.

7. Pascual-Pascual SI, Nascimento A, Fernández-Llamazares CM Medrano-López C, Villalobos-Pinto E, Martínez-Moreno M et al. Guía clínica de la enfermedad de Pompe infantil. Rev Neurol 2016; 63: 269-79.

8. Kazi ZB, Desai AK, Berrier KL, Troxler RB, Wang RY, AbdulRahman OA, et al. Sustained immune tolerance induction in enzyme replacement therapy-treated CRIM-negative patients with infantile Pompe disease. JCI Insight 2017; 2: e94328.

9. Banugaria SG, Prater SN, Patel TT, Dearmey SM, Milleson C, Sheets KB, et al. Algorithm for the early diagnosis and treatment of patients with cross reactive immunologic materialnegative classic infantile Pompe disease: a step towards improving the efficacy of ERT. PLoS One 2013; 8: e67052.

10. Banugaria SG, Patel TT, Kishnani PS. Immune modulation in Pompe disease treated with enzyme replacement therapy. Expert Rev Clin Immunol 2012; 8: 497-9.

11. Howell RR, Byrne B, Darras BT, Kishnani P, Nicolino M, Van der Ploeg A. Diagnostic challenges for Pompe disease: an under-recognized cause of floppy baby syndrome. Genet Med 2006; 8: 289-96.

\section{A new phenotype of infantile-onset Pompe disease}

Introduction. Infantile-onset Pompe disease is a kind of glycogenosis resulting from a deficit of the enzyme acid alphaglucosidase. Before specific enzyme replacement therapy (ERT) became available, the classic form was fatal during the first two years of life. ERT increases survival and improves cardiac, respiratory and motor functioning.

Case reports. Case 1: 2-month-old infant with predominantly axial hypotonia who required the use of a nasogastric tube as a result of difficulties in sucking and swallowing. Myopathy and hypertrophic cardiomyopathy were observed. The patient was diagnosed with Pompe disease, ERT was established and improved heart and motor functioning were noted. Nevertheless, she presented recurring respiratory infections that finally made it necessary to perform a tracheostomy. She is currently still undergoing ERT, walks with a walker and presents a mild ventricular dysfunction. Case 2: 3-week-old infant who was taken to see his paediatrician for a routine check-up. The examination revealed a systolic bruit and axial and proximal hypotonia. Cardiology tests revealed hypertrophic cardiomyopathy. The patient was sent to a referral centre, where he was diagnosed with Pompe disease and a cross-reactive immunological material-negative status. The patient received immunomodulator treatment and ERT. Progress was favourable, although he presented frequent respiratory infections. The patient is currently capable of walking by himself, although the gait is unsteady.

Conclusions. Both cases illustrate the new phenotype of infantile-onset Pompe disease treated with ERT. Despite the motor limitations and respiratory involvement presented by the patients, both survival and autonomy have increased.

Key words. Acid alpha-glucosidase. CRIM status. Enzyme replacement therapy. Hypertrophic cardiomyopathy. Hypotonia. Pompe disease. 